\title{
A New and General Method for Blind Shift-Variant Deconvolution of Biomedical Images
}

\author{
Moritz Blume ${ }^{1}$, Darko Zikic ${ }^{1}$, Wolfgang Wein ${ }^{1,2}$, and Nassir Navab ${ }^{1}$ \\ ${ }^{1}$ Computer Aided Medical Procedures (CAMP), Technische Universität München, Germany \\ $\{$ blume, zikic, navab\}@cs. tum. edu \\ ${ }^{2}$ Siemens Corporate Research, Princeton, USA \\ wolfgang.wein@siemens.com
}

\begin{abstract}
We present a new method for blind deconvolution of multiple noisy images blurred by a shift-variant point-spread-function (PSF). We focus on a setting in which several images of the same object are available, and a transformation between these images is known. This setting occurs frequently in biomedical imaging, for example in microscopy or in medical ultrasound imaging. By using the information from multiple observations, we are able to improve the quality of images blurred by a shift-variant filter, without prior knowledge of this filter. Also, in contrast to other work on blind and shift-variant deconvolution, in our approach no parametrization of the PSF is required. We evaluate the proposed method quantitatively on synthetically degraded data as well as qualitatively on $3 \mathrm{D}$ ultrasound images of liver. The algorithm yields good restoration results and proves to be robust even in presence of high noise levels in the images.
\end{abstract}

\section{Introduction}

Biomedical imaging techniques suffer - like all measurements - from errors introduced in the acquisition process. Techniques which are able to remove these errors can improve the quality of the images and thus enhance their diagnostic value. Since blur is one of the most common image degradations, deconvolution methods which undo the effects of blurring and thus reveal structures not visible in the uncorrected images are extremely important in biomedical imaging. For medical imaging devices such as ultrasound or microscopy, especially the case in which the blur is not constant in the image the so-called shift-variant blur - is important. The case of constant (shift-invariant) blur hardly occurs in biomedical applications.

The problem of deconvolution of shift-variantly blurred images is an extremely challenging task. One part of the difficulty is to determine the point-spread-function (PSF), that is, the function characterizing the blur. Up to now, this is achieved by either theoretical predictions (see e. g. [1] for medical ultrasound) or measurements. Both have to be performed for every single device and are time-consuming, complicated, error-prone, and in some cases even not feasible. Deconvolution methods that build on this previous step can not be considered as general.

As a remedy, most of the existing work assumes a simplified model, the shift-invariant one. This is tempting since it allows for the very attractive option of restoring both the image and the PSF within one method, without the need of measuring or theoretically

N. Ayache, S. Ourselin, A. Maeder (Eds.): MICCAI 2007, Part I, LNCS 4791, pp. 743-750, 2007.

(C) Springer-Verlag Berlin Heidelberg 2007 
predicting the PSF. This class of methods is denoted as blind deconvolution methods. Unfortunately, assuming a shift-invariant model very often comes at the price of not adecuately describing biomedical reality. Therefore, in many cases such methods do not yield optimal restorations. It would be very attractive to have both: the shift-variant model and a blind restoration method able to deal with this model.

In this paper, we present such a blind and shift-variant method. Our method uses multiple images of the same object together with the information about the transformation between the images. This is a setting readily available in many medical and microscopic applications, and, thanks to advancing image registration techniques (especially deformable registration), will be more and more widespread in the future. Since in this setting the observations are blurred by the same PSF, each additional observation provides information about the present degradation. Our method takes this information into account for the restoration of both the image and the PSF.

To the best of our knowledge, this is the first method capable of performing an unparameterized, blind, and shift-variant deconvolution. These properties make our method extremely general and widely and easily applicable to many applications in the biomedical imaging field.

\subsection{Related Work}

Single-view deconvolution is a standard post-processing procedure in biomedical applications such as ultrasound [2] or microscopy [3]. Unfortunately, restoration from a single-view is a dramatically ill-posed problem, and in the best case allows for blind shift-invariant deconvolution in a low noise scenario. In photography, several semi-blind shift-variant methods have been proposed [4[56]7|8]. However, these methods rely on a priori information about the blur, e. g. that the PSF is an instance of motion blur or outof-focus blur, and thus can not be considered as general. For microscopy, some dedicated methods are known: in [9], the volume subject to restoration is reconstructed block-wise, and shift-invariance is assumed for each block. Preza and Conchello [10] assume to know the PSF at several points in space and restrict shift-variance to the depth direction.

Already in the 1970's researchers found that the multi-view scenario has advantages over single-view restoration, since each additional observation adds information that can be taken into account for the restoration process [11]. Since then, especially dedicated devices for this multi-view setting have been developed for both microscopy and ultrasound [12 13, 14[15]16]. Existing restoration methods from the single-view setting have been adapted and new methods especially dedicated to the multi-view setting have been invented. Shaw et al. [17] combine several blurred observations by taking the maximum in Fourier space. Their restoration is fast and simple to implement. However it is not optimal, even for synthetic images, and also dramatically amplifies noise. Kikuchi et al. calculate synthetic projections from two blurred observations and then use those projections that are less affected by blur for a standard computed tomography reconstruction algorithm [18]. Though being well suited for their specific scenario, this is not quite general since it only works for the shift-invariant case and the direction of the blur has to be known in advance. Often, very simple restoration techniques like linear combination are applied and are reported to lead to a significant improvement of the 
resolution [14 15]. Soler et al. propose a non-blind shift-invariant deconvolution method for ultrasound that relies on a measured PSF [16].

\section{Method}

\subsection{Model}

In contrast to other multi-view techniques, we use the shift-variant image formation model

$$
z_{i}=\left(u \circ \varphi_{i}\right) \star h+\eta
$$

where $z_{i} \in F$ is the actual measurement ( $F$ denotes the functional space of all images $\Omega \rightarrow \mathbb{R}$ where $\Omega$ is a bounded domain $\left.\Omega=[0,1]^{2} \subset \mathbb{R}^{2}\right), u \in F$ is the original image, $\varphi_{i}: \Omega \rightarrow \Omega$ is the spatial transformation between measurement and original image, $h:(\Omega \times \Omega) \rightarrow \mathbb{R}$ is the shift-variant PSF, " $\star$ " is the shift-variant convolution operator and $\eta \in F$ represents arbitrary noise. Using this model, we assume that the PSF is equal for each observation, that is, not changing over time. This is a valid assumption for most biomedical applications. Please note that we do not restrict the transformation $\varphi$ to rigid transformations which implies that also deformations are within our model.

In presence of noise, we can not assume that $z_{i}=u\left(\varphi_{i}\right) \star h$ holds exactly, so we formulate the cost function which is subject to minimization as

$$
\mathcal{D}[u, h]=\frac{1}{2} \sum_{i=1}^{P} \int_{\Omega_{i}} e_{i}(\mathbf{x})^{2} d \mathbf{x},
$$

where we suppose to dispose of $P$ measurements. $e_{i}(\mathbf{x})$ is an error term that measures the difference of the convolved real image to the measurements at position $\mathbf{x}$ by

$$
e_{i}(\mathbf{x})=\left(\left(u \circ \varphi_{i}\right) \star h-z_{i}\right)(\mathbf{x})=\int_{\Omega} u\left(\varphi_{i}(\xi)\right) h(\mathbf{x}, \xi-\mathbf{x}) d \xi-z_{i}(\mathbf{x}) .
$$

The set $\Omega_{i}$ denotes the overlapping domain of the original image $u$ and the image transformed by $\varphi_{i}$.

The problem of minimizing the functional from Equation (2) is ill-posed. In order to render the problem well-posed we introduce regularizing terms $\mathcal{U}$ and $\mathcal{H}$. While $\mathcal{U}$ operates on the image $u$, the term $\mathcal{H}$ imposes restrictions on the point-spread-function $h$.

So we define the regularized functional $\mathcal{I}: F \times F \rightarrow \mathbb{R}$ to be minimized which models the problem as

$$
\mathcal{J}[u, h]=\mathcal{D}[u, h]+\alpha \mathcal{U}[u]+\beta \mathcal{H}[h] .
$$

Here, the positive real coefficients $\alpha$ and $\beta$ determine the influence of the respective regularization term.

Additionally, since we assume to deal with an intensity PSF, we restrict $h$ to zero and positive values, that is, $h(\mathbf{x}, \mathbf{s}) \geq 0$. We also assume that the imaging system neither emits nor absorbs energy and so the PSF has to be normed to one at every spatial 

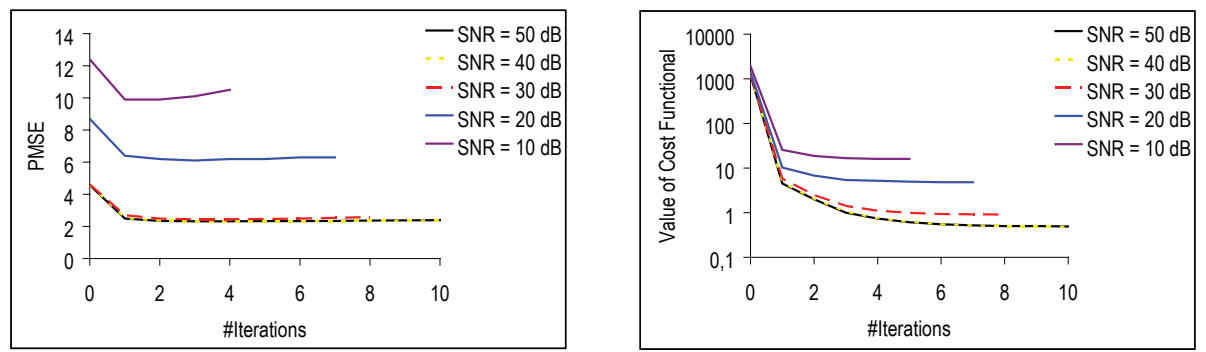

Fig. 1. The percentage mean squared error (PMSE, left plot) and the cost functional from equation (4) (right plot) are plotted over the iterations for the hippocampal rat neurons example - compare Figure (2). For low noise levels, the PMSE is reduced to approximately half of its initial value. Even for extremely noisy images our method provides a significant reduction of the PMSE. The cost function is reduced for each iteration and the algorithm terminates when its reduction is stagnating.

position $\mathbf{x}$ in the image, that is $\int_{\Omega} h(\mathbf{x}, \mathbf{s}) d \mathbf{s}=1$. The final goal then is to find functions $u$ and $h$ that minimize $\mathcal{I}$ subject to constraints, that is, compute

$$
\underset{u, h}{\operatorname{argmin}} J[u, h] \quad \text { subject to } h(\mathbf{x}, \mathbf{s}) \geq 0 \text { and } \int_{\Omega} h(\mathbf{x}, \mathbf{s}) d \mathbf{s}=1 \quad \forall \mathbf{x}, \mathbf{s} \in \Omega .
$$

Regularization Term $\mathcal{U}$. Total variation [19] has proven to be a very successful method for image regularization since it discourages noise while edges are preserved

$$
\mathcal{U}[u]=\int_{\Omega}\left|\nabla_{\mathbf{x}} u(\mathbf{x})\right| d \mathbf{x} .
$$

This regularization term penalizes too large gradients in the image to be reconstructed.

Regularization Term $\mathcal{H}$. As mentioned, the lack of information due to the shift-variant PSF is tremendous. In order to account for this lack of information and the resulting ill-posedness of the problem, we introduce a regularization term $\mathcal{H}$ which operates on the PSF:

$$
\mathcal{H}[h]=\iint_{\Omega}\left|\nabla_{\mathbf{x}} h(\mathbf{x}, \mathbf{s})\right|^{2} d \mathbf{s} d \mathbf{x} .
$$

A shift-variant PSF can be considered as a set of shift-invariant PSFs for each pixel x. The regularization term $\mathcal{H}$ penalizes the difference between the shift-invariant PSFs at neighboring pixels. It is important to note that this regularization will not impose smoothness on the PSF itself but only on their transitions.

\subsection{Algorithm}

The main difficulty of blind deconvolution in general is that we neither have $u$ nor $h$. For this type of problem, You and Kaveh identify a scale problem [20] and so justify an algorithmic scheme that is alternating between the calculation of $h$ and $u$. For the 

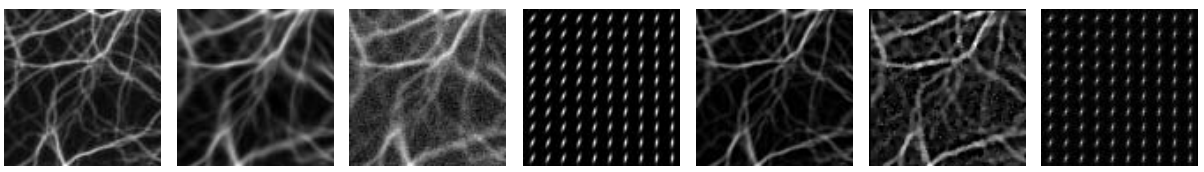

Fig. 2. The original image of hippocampal rat neurons (left) is blurred by a shift-variant PSF (fourth from left) and noise of different strength is added (second and third). Only the first of four observations for each noise case is shown. The restored images and the restored PSF for the 50 $\mathrm{dB}$ noise case are shown aside. For 2D images, a shift-variant PSF can be considered as a 2D grid of shift-invariant PSFs for each pixel. For the sake of a clear visualization, the PSF is only imaged for a region of interest of $10 \times 10$ image pixels.

calculation of $h$ we assume that $u$ is given, and for the calculation of $u$ we assume $h$ to be given. These two steps are executed alternately until convergence

$$
\begin{array}{ll}
\text { First step: } & h \leftarrow \underset{h}{\operatorname{argmin}} \mathcal{I}[u, h] \text { subject to constraints on } h \\
\text { Second step: } & u \leftarrow \underset{u}{\operatorname{argmin}} \mathcal{I}[u, h] .
\end{array}
$$

These two optimization problems are treated completely independently. In first step, we assume that $u$ is perfectly known and on this base $h$ is sought. As an initial guess for $u$ we take the first observation $z_{1}$. After discretization, any constrained least-squares optimizer can be used. In the second optimization problem we assume that $h$ is perfectly known and on this base $u$ is sought. We derive the Euler-Lagrange first order optimality equation corresponding to this optimization problem by extending the derivation of [8] to the multi-view scenario:

$$
\sum_{i} \int_{\Omega_{i}} e_{i}\left(\xi+\varphi_{i}^{-1}(\mathbf{x})\right) h\left(\xi+\varphi_{i}^{-1}(\mathbf{x}), \xi\right) d \xi=-\alpha \nabla_{\mathbf{x}}^{\top} \frac{1}{\sqrt{u_{x_{1}}^{2}+u_{x_{2}}^{2}+\varepsilon^{2}}} \nabla_{\mathbf{x}} u .
$$

The image which is subject to reconstruction is found by solving this PDE.

We discretize equation (10) with implicit finite-differences. This leads to a nonlinear system of equations. The nonlinearity can be faced by rearranging it as a fixed-point equation which leads to an iterative solution scheme. Each iteration then involves the solution of a very large linear system of equations that can be solved by any standard method. We use the method of conjugate gradients.

\section{Results}

\subsection{Quantitative Evaluation}

We use a simulation of the degradation process with known ground truth. We evaluate our method for several test cases with different noise levels. In each case, four observations are synthetically generated by transforming and degrading an original image. We simulate the image acquisition process by a device suffering from shift-variant blurring and noise. For the simulation, we perform the following steps for each observation: 

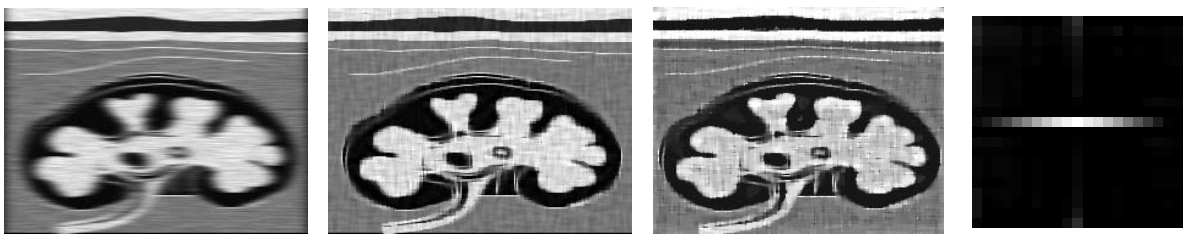

Fig. 3. Comparison of our blind shift-variant method to Soler's non-blind shift-invariant method: Soler et al. simulate a multi-view setting by degrading a synthetic image of the kidney with shiftinvariant Gaussian blur and noise. Only the first of two generated observations is shown (left). For their restoration in (second from left), they use the known PSF. Our method restores both the image and the PSF (third and fourth from left), without any a priori knowledge about the PSF. Even so, our results are comparable.

(i) rotation and translation, (ii) blurring (simulated by shift-variant filtering) and finally (iii) addition of white Gaussian noise. The test cases differ in the amount of added noise. We measure the noise level by the signal to noise ratio SNR $=P_{\mathbf{z}_{i}} / P_{\text {Noise }}$, where the signal power $P_{\mathbf{z}_{i}}$ is the power of the observations $\mathbf{z}_{i}$ and $P_{\text {Noise }}$ is the noise power. The quality of reconstruction for the image is measured by the percentage mean squared error $\mathrm{PMSE}_{\mathbf{u}}=\left|\mathbf{u}-\mathbf{u}^{\text {orig }}\right|^{2} /\left(|\Omega|\left|\mathbf{u}^{\text {orig }}\right|^{2}\right)$.

For low noise levels, Figure 2 shows clearly that the obtained restoration of both the image and the PSF comes close to their respective originals. Our restoration leads to better defined structures even for very high noise levels. The reduction of the PMSE as well as the evaluation of the cost functional of equation (4) is presented in Figure 1 . We observe fast and robust convergence of the algorithm for all test cases.

\subsection{D Ultrasound Restoration of a Volunteer's Liver}

We apply our algorithm to a 3D ultrasound acquisition of a volunteer's liver acquired with the Siemens SONOLINE Antares (c). We acquire two volumes of size of $100^{3}$ voxels each, and the second volume is rotated by an angle of approximately $90^{\circ}$ about the axial direction. Figure 4 shows selected slices of different orientations of the first observation. The corresponding restoration disposes of better defined structures and reveals some details not quite visible in the observation. Note that the blur extension of the restored PSF varies spatially. Our algorithm performs five iterations until convergence and in our current $\mathrm{C}++$ implementation this takes about six hours.

\subsection{Comparison to a Non-blind Shift-Invariant Method}

Comparison of our method to other deconvolution methods is a delicate matter, since - to the best of our knowledge - there is no method that performs blind shift-variant deconvolution without assuming an a priori blur model. However, we compare our method to the recently proposed non-blind shift-invariant method of Soler et al. [16]. There, synthetic images of a kidney are blurred by shift-invariant Gaussian blur and noise is added and the known PSF is used for restoration. Figure 3 shows that though our method does not use the PSF and assumes a shift-variant blur, it produces competitive results. It restores both the image and the PSF. The restored PSF fits to the specifications made in [16]. 

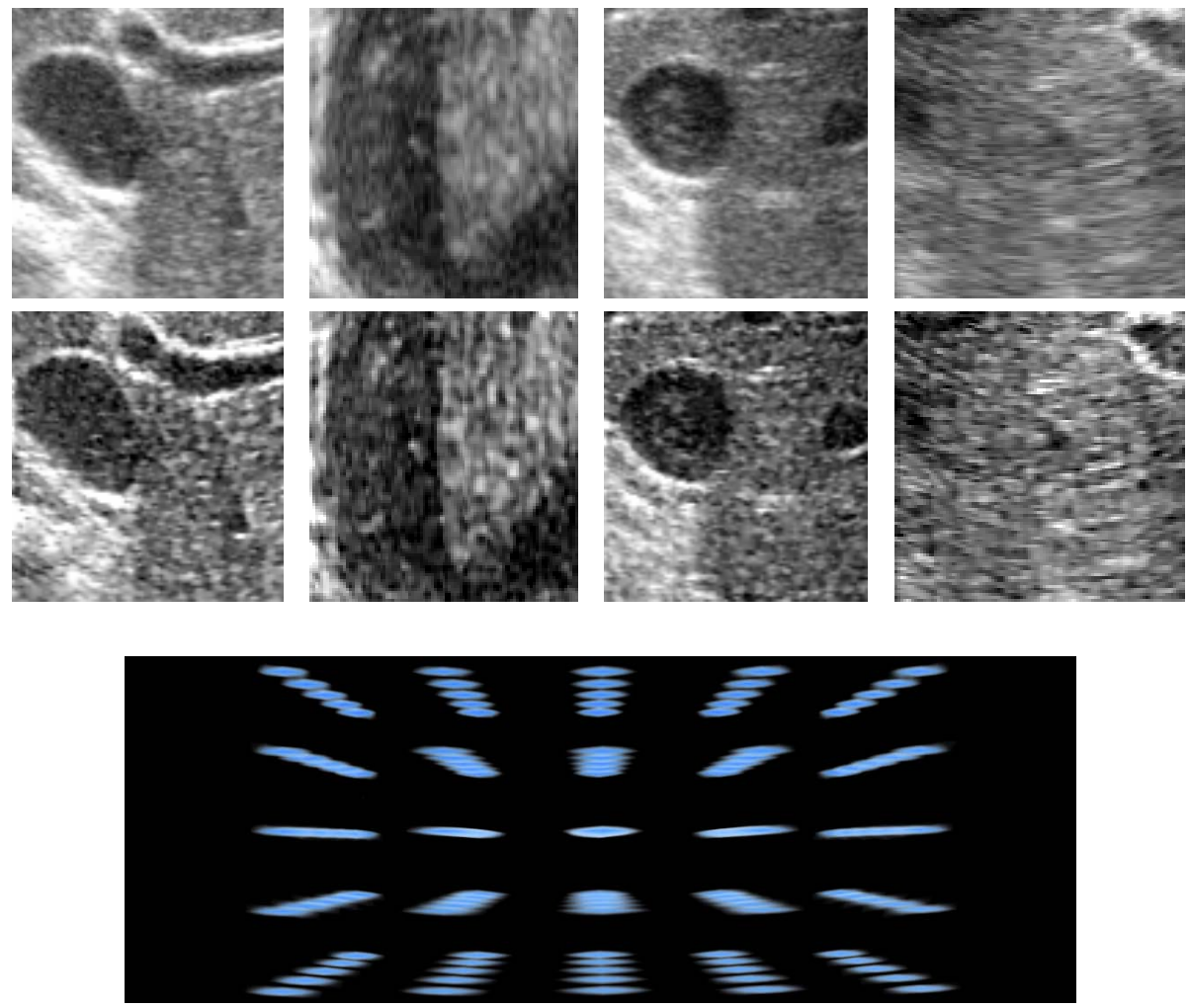

Fig. 4. Multi-view unparameterized blind shift-variant deconvolution of 3D ultrasound acquisitions of a volunteer's liver: two 3D spatially transformed observations are used for restoration. The transformation between the two observations is obtained by deformable registration as described in [21]. The first row shows selected slices of the first observation. The corresponding restored slices are shown in the second row. Last row: restored PSF. For 3D images, a shiftvariant PSF can be considered as a 3D grid of shift-invariant PSFs for each voxel (for the sake of a clear visualization we use a coarser grid). It can be clearly seen that the PSF is changing its shape from the lower layers to the upper layers.

\section{Conclusion}

We present a new method for multi-view blind deconvolution. Existing blind methods assume a stationary blur that is not changing over the image. In contrast, we use a shiftvariant blur model that allows the blur to vary with the spatial position in the image. This makes our method better suited for biomedical applications than shift-invariant methods. Due to its blind character, there is no need for previous measurements or theoretical predictions of the PSF. We show experimentally that our method is even competitive to non-blind shift-invariant methods. Our experiments prove robustness, also for extremely high noise levels. 


\section{References}

1. Ng, J., Prager, R., Kingsbury, N., Treece, G., Gee, A.: Modeling ultrasound imaging as a linear, shift-variant system. Ultrasonics, Ferroelectrics and Frequency Control, IEEE Transactions on 53(3), 549-563 (2006)

2. Jirik, R., Taxt, T.: Two-dimensional blind iterative deconvolution of medical ultrasound images. In: Ultrasonics Symposium, IEEE Computer Society Press, Los Alamitos (2004)

3. Sarder, P., Nehorai, A.: Deconvolution methods for 3-d fluorescence microscopy images. IEEE Signal Processing Magazine 23(3), 32-45 (2006)

4. Sawchuk, A.: Space-variant image motion degradation and restoration. Proceedings of the IEEE 60(7), 854-861 (1972)

5. Tekalp, A.M., Kaufman, H., Woods, J.W.: Model-based segmentation and space-variant restoration of blurred images by decision-directed filtering. Signal Process. 15(3), 259-269 (1988)

6. Lagendijk, R.L., Biemond, J.: Block-adaptive image identification and restoration. In: Acoustics, Speech, and Signal Processing, 1991. ICASSP 1991. 1991 International Conference on, Toronto, Ont. Canada pp. 2497-2500 (1991)

7. Trussell, H., Fogel, S.: Identification and restoration of spatially variant motion blurs in sequential images. IEEE Transactions on Image Processing 1(1), 123-126 (1992)

8. You, Y.L., Kaveh, M.: Blind image restoration by anisotropic regularization. Image Processing, IEEE Transactions on 8(3), 396-407 (1999)

9. Avinash, G.B.: Data-driven, simultaneous blur and image restoration in 3-d fluorescence microscopy. Journal of Microscopy 183(2), 145-157 (1996)

10. Preza, C., Conchello, J.A.: Depth-variant maximum-likelihood restoration for threedimensional fluorescence microscopy. J. Opt. Soc. Am. A 21, 1593-1601 (2004)

11. Skaer, R., Whytock, S.: Interpretation of the three-dimensional structure of living nuclei by specimen tilt. J. Cell. Sci. 19(1), 1-10 (1975)

12. Kikuchi, S., Sonobe, K., Shinohara, D., Shinro Mashiko, N.O., Hiraoka, Y.: A double-axis microscope and its three-dimensional image position adjustment based on an optical marker method. Optics Communications 129, 237-244 (1996)

13. Kozubek, M., Matula, P., Eipel, H., Hausmann, M.: Automated multi-view 3d image acquisition in human genome research. In: 3D Data Processing Visualization and Transmission. In: Proceedings. First International Symposium on pp. 91-98 (2002)

14. Swoger, J., Huisken, J., Stelzer, E.H.K.: Multiple imaging axis microscopy improves resolution for thick-sample applications. Optics Letters 28, 1654-1656 (2003)

15. Huisken, J., Swoger, J., Bene, F.D., Wittbrodt, J., Stelzer, E.H.K.: Optical sectioning deep inside live embryos by selective plane illumination microscopy. Science 305(5686), 10071009 (2004)

16. Soler, P., Villain, N., Bloch, I., Angelini, E.: Volume reconstruction of breast echography from anisotropically degradated scans. In: Proceedings of the IASTED International Conference on Biomedical Engineering, vol. 9, pp. 349-355 (2005)

17. Shaw, P.J., Agard, D.A., Hiroaka, Y., Sedat, J.W.: Tilted view reconstruction in optical microscopy. Biophysical Journal 55, 101-110 (1989)

18. Kikuchi, S., Sonobe, K., Sidharta, L.S., Ohyama, N.: Three-dimensional computed tomography for optical microscopes. Optics Communications 107, 432-444 (1994)

19. Rudin, L.I., Osher, S., Fatemi, E.: Nonlinear total variation based noise removal algorithms. Physica D 60, 259-268 (1992)

20. You, Y.L., Kaveh, M.: A regularization approach to joint blur identification and image restoration. IEEE Transactions on 5(3), 416-428 (1996)

21. Zikic, D., Wein, W., Khamene, A., Clevert, D.A., Navab, N.: Fast deformable registration of 3D-ultrasound using a variational approach. In: Larsen, R., Nielsen, M., Sporring, J. (eds.) MICCAI 2006. LNCS, vol. 4190, Springer, Heidelberg (2006) 